\title{
Gaussian Effective Potential and the Coleman's normal-ordering Prescription : the Functional Integral Formalism
}

\author{
Wen-Fa Lu * and Chul Koo Kim \\ Institute of Physics and Applied Physics, Yonsei University, Seoul 120-749, Korea \\ Center for Strongly Correlated Materials Research, Seoul National University, Seoul 151-742, Korea
}

\begin{abstract}
For a class of system, the potential of whose Bosonic Hamiltonian has a Fourier representation in the sense of tempered distributions, we calculate the Gaussian effective potential within the framework of functional integral formalism. We show that the Coleman's normal-ordering prescription can be formally generalized to the functional integral formalism.
\end{abstract}

Recently, one of the authors, Lu, with his other collaborators obtained formulae of the Gaussian effective potential (GEP) [1] for a relatively general scalar field theory (see Eq.(1)) in the functional Schrödinger picture [2]. There, 'the Coleman's normal-ordering prescription [3] was used, and accordingly these formulae have no divergences in low dimensions. Employing these formulae, one can obtain the GEP of any system in a certain class of models, which will 'be specified below, by carrying out ordinary integrations without performing functional integrations. In this paper, 'we demonstrate that the same formulae of the GEP can also be obtained within the functional integral formalism. In 'doing so, we also show that, although quantities in the functional integral formalism are not operators, the Coleman's 'normal-ordering prescription can be formally used for renormalizing the GEP in the cases of low dimensions. We believe that our simple work is interesting and useful, since the functional integral formalism is importmant in quantum

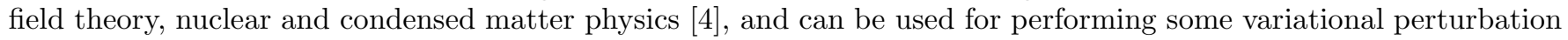
schemes [5,6].

In this paper, we first generalize the Coleman's normal-ordering prescription to the functional integral formalism. This formal generalization will be realized by borrowing the normal-ordered Hamiltonian expression in the functional 'Schrödinger picture because the Euclidean action for a system has the same form with the corresponding classical 'Hamiltonian in the Minkowski space. Then, following the procedure in Ref. [6], we calculate the GEP for a class of 'systems. Finishing the above generalization, as an explicit illustration, we will perform a model calculation for the $\lambda \phi^{4}$ field theory.

Consider a class of systems, scalar field systems or Fermi field systems which can be bosonized, with the Lagrangian 'density

$$
\mathcal{L}_{x}=\frac{1}{2} \partial_{\mu} \phi_{x} \partial^{\mu} \phi_{x}-V\left(\phi_{x}\right)
$$

where the subscript $x$ represents, $x=(\vec{x}, t)$, the coordinates in a $(D+1)$-dimensional Minkowski space, $\partial_{\mu}$ and $\partial^{\mu}$ are the corresponding covariant derivatives, and $\phi_{x}$ the scalar field at $x$. In Eq.(1), the potential $V\left(\phi_{x}\right)$ has a 'Fourier reprensentation in a sense of tempered distributions [7]. Speaking roughly, this requires that the integral $\int_{-\infty}^{\infty} V(\alpha) e^{-C \alpha^{2}} d \alpha$ with a positive constant $C$ is finite. Obviously, quite a number of model potentials, such as polynomial models, sine-Gordon and sinh-Gordon models, possess this property.

For the system, Eq.(1), the conjugate field momentum is expressed as $\Pi_{x} \equiv \frac{\partial \mathcal{L}}{\partial\left(\partial_{t} \phi_{x}\right)}=\partial_{t} \phi_{x}$, and the Hamiltonian density is given by

$$
\mathcal{H}_{x}=\frac{1}{2} \partial_{t} \phi_{x} \partial_{t} \phi_{x}+\frac{1}{2} \partial_{\vec{x}} \phi_{x} \partial_{\vec{x}} \phi_{x}+V\left(\phi_{x}\right)
$$

In a time-fixed functional Schrödinger picture at $t=0$, one can normal-order the Hamiltonian density $\mathcal{H}_{x}$ with respect to any given mass-dimension constant $M$ as follows [2, 3] 1 :

$$
\mathcal{N}_{M}\left[\mathcal{H}_{\vec{x}}\right]=\frac{1}{2} \partial_{t} \phi_{x} \partial_{t} \phi_{x}+\frac{1}{2} \partial_{\vec{x}} \phi_{\vec{x}} \partial_{\vec{x}} \phi_{\vec{x}}+\mathcal{N}_{M}\left[V\left(\phi_{\vec{x}}\right)\right]-\frac{1}{2} I_{0}\left[M^{2}\right]+\frac{1}{4} M^{2} I_{1}\left[M^{2}\right],
$$

\footnotetext{
*Permanent address: Department of Physics, and Institute for Theoretical Physics, Shanghai Jiao Tong University, Shanghai 200030, the People's Republic of China; E-mail: wenfalu@online.sh.cn

${ }^{1}$ Here, the partial derivative with the time $\partial_{t} \phi_{x}=\left.\left(\partial_{t} \phi_{x}\right)\right|_{t=0}$ should be regarded as the conjugate momentum operator $\Pi_{\vec{x}}$. For convenience of later comparison, we write the operator as its corresponding classical form.
} 
where, $\mathcal{N}_{M}[\cdots]$ means the normal-ordering form with respect to $M$ and

$$
I_{n}\left[Q^{2}\right]=\int \frac{d^{D} p}{(2 \pi)^{D}} \frac{\sqrt{p^{2}+Q^{2}}}{\left(p^{2}+Q^{2}\right)^{n}} .
$$

Noticing the Baker-Haussdorf formula $e^{A+B}=e^{A} e^{B} e^{-\frac{1}{2}[A, B]}$, with the commutator $[A, B]$ some c-number, one has

$$
\mathcal{N}_{M}\left[V\left(\phi_{\vec{x}}\right)\right]=\int \frac{d \Omega}{\sqrt{2 \pi}} \tilde{V}(\Omega) e^{i \Omega \phi_{\vec{x}}} e^{\frac{1}{4} \Omega^{2} I_{1}\left[M^{2}\right]}
$$

where $\tilde{V}(\Omega)$ is the Fourier component of the function $V(\alpha)$. In the functional Schrödinger picture, when the Coleman's normal-ordering prescription [3] is adopted to calculate the GEP, i.e., when the normal-ordering Hamiltonian density takes the place of Hamiltonian density, the GEP will be naturally finite for the case of low dimensions $(D<3)$ [2].

On the other hand, for Eq.(1), the Generating functional for Green's functions in the functional integral formalism is given by

$$
Z_{M}[J]=\int \mathcal{D} \phi \exp \left\{i \int d^{D} \vec{x} d t\left[\mathcal{L}_{x}+J_{x} \phi_{x}\right]\right\}
$$

where, $J_{x}$ is an external source at $x$, and $\mathcal{D} \phi$ the functional measure. Starting from Eq.(6), one can define the effective potential [8]. However, in this functional integral, the integrand is oscillatory. To avoid this oscillation, one usually adopts the so-called $\epsilon$-prescription, that is, in the Lagrangian density, one adds an infinitesimal purely imaginary term, $i \epsilon \phi_{x}^{2}$, and takes $\epsilon \rightarrow 0$ after finishing the functional integration. Instead of doing so, one can also make the time continuation $t \rightarrow-i \tau$ so that the Minkowski space can be transformed into the Euclidean space, and after finishing the functional integration, one may return to the Minkowski space. This time continuation procedure is equivalent to the $\epsilon$-prescription [8]. In this paper, we will choose the continuation procedure.

Through the time continuation $t \rightarrow-i \tau$, the generating functional $Z_{M}[J]$ is changed as

$$
Z[J]=\int \mathcal{D} \phi \exp \left\{-\int d^{\nu} r\left[\frac{1}{2} \partial_{\tau} \phi_{r} \partial_{\tau} \phi_{r}+\frac{1}{2} \partial_{\vec{x}} \phi_{r} \partial_{\vec{x}} \phi_{r}+V\left(\phi_{r}\right)-J_{r} \phi_{r}\right]\right\}
$$

with $r=(\vec{x}, \tau)$ and $\nu=D+1$. This is the generating functional in the Euclidean space. Here, we emphasize that in the above equation, taking the range of $\tau$ as $[0, \beta]$ with the inverse temperature $\beta$, letting $J_{r}$ vanish and carrying out the functional integration over the closed path $\left.\phi_{r}\right|_{\tau=0}=\left.\phi_{r}\right|_{\tau=\beta}$, one can arrive at the canonical partition function of Eq.(1). From Eq.(7), one can get the generating functional for the connected Green's function, $W[J]=\ln (Z[J])$. The variational derivative of $W[J]$ with respect to $J$ will give rise to the vacuum expectation value of the field $\phi_{r}$ in the presence of $J_{r}$

$$
\varphi_{r}=\frac{\delta W[J]}{\delta J_{r}} .
$$

Taking a Legendre transformation of $W[J]$, one can define the effective potential in Euclidean space,

$$
\mathcal{V}(\varphi)=-\left.\frac{W[J]-\int d^{\nu} r J_{r} \varphi_{r}}{\int d^{\nu} r}\right|_{\varphi_{r}=\varphi},
$$

where $\varphi$ is independent of the coordinate $r$. Returning to the Minkowski space from Eq.(9), one can get the effective potential in the Minkowski space, which is usually refered to as effective potential in quantum field theory.

In the exponential of the functional integrand of Eq.(7), the major part of the integrand $\mathcal{H}_{r}=\frac{1}{2} \partial_{\tau} \phi_{r} \partial_{\tau} \phi_{r}+$ $\frac{1}{2} \partial_{\vec{x}} \phi_{r} \partial_{\vec{x}} \phi_{r}+V\left(\phi_{r}\right)$ takes the same form of $\mathcal{H}_{x}$ in Eq.(2). Therefore, we argue that, if we change $\mathcal{H}_{r}$ in Eq.(7) into the expression of $\mathcal{N}_{M}\left[\mathcal{H}_{r}\right]$ 2 the GEP will be renormalized automatically in the low dimensions $(D<3)$. Note that in the transformation between the Euclidean space and the Minkowski space, the integrals $I_{(n)}\left[Q^{2}\right]$ appearing in the functional integrations in the Euclidean space

\footnotetext{
${ }^{2}$ Note that $\mathcal{H}_{x}$ in Eq.(3) is an operator in D-dimensional space at $t=0$, whereas the field $\phi_{r}$ and its derivatives in Eq.(7) are classical ones in $\nu$-dimensional Euclidean space. This is why we call the generalization developed in the present paper as a formal generalization.
} 


$$
I_{(n)}\left[Q^{2}\right]= \begin{cases}\int \frac{d^{\nu} p}{(2 \pi)^{\nu}} \frac{1}{\left(p^{2}+Q^{2}\right)^{n}}, & \text { for } n \neq 0 \\ \int \frac{d^{\nu} p}{(2 \pi)^{\nu}} \ln \left(p^{2}+Q^{2}\right), & \text { for } n=0\end{cases}
$$

are equivalent to $I_{n}\left[Q^{2}\right]$ in Eq.(4) which appear in the calculations in functional Schrödinger picture (up to some constant factor or an infinite constant for some $n$ ) [6]. For example, $I_{(0)}\left[Q^{2}\right]$ is equivalent to $I_{0}\left[Q^{2}\right]$ (up to an infinite constant) and $2 I_{(1)}\left[Q^{2}\right]$ to $I_{1}\left[Q^{2}\right][6]$. Thus, corresponding to Eq.(3), one can formally write down $\mathcal{N}_{M}\left[\mathcal{H}_{r}\right]=$ $\frac{1}{2} \partial_{\tau} \phi_{r} \partial_{\tau} \phi_{r}+\frac{1}{2} \partial_{\vec{x}} \phi_{r} \partial_{\vec{x}} \phi_{r}+\mathcal{N}_{M}\left[V\left(\phi_{r}\right)\right]-\frac{1}{2} I_{(0)}\left[M^{2}\right]+\frac{1}{2} M^{2} I_{(1)}\left[M^{2}\right]$. Changing $\mathcal{H}_{r}$ in Eq. (7) into the form of $\mathcal{N}_{M}\left[\mathcal{H}_{r}\right]$, we have

$$
\begin{aligned}
Z[J]= & \exp \left\{\int d^{\nu} r\left[\frac{1}{2} I_{(0)}\left[M^{2}\right]-\frac{1}{2} M^{2} I_{(1)}\left[M^{2}\right]\right]\right\} \int \mathcal{D} \phi \exp \left\{-\int d^{\nu} r\left[\frac{1}{2} \partial_{\tau} \phi_{r} \partial_{\tau} \phi_{r}+\frac{1}{2} \partial_{\vec{x}} \phi_{r} \partial_{\vec{x}} \phi_{r}\right.\right. \\
& \left.\left.-J_{r} \phi_{r}+\int \frac{d \Omega}{\sqrt{2 \pi}} \tilde{V}(\Omega) e^{i \Omega \phi_{r}} e^{\frac{1}{2} \Omega^{2} I_{(1)}\left[M^{2}\right]}\right]\right\} \\
= & \exp \left\{\int d^{\nu} r\left[\frac{1}{2} I_{(0)}\left[M^{2}\right]-\frac{1}{2} M^{2} I_{(1)}\left[M^{2}\right]\right]\right\} \int \mathcal{D} \phi \exp \{-S[J]\},
\end{aligned}
$$

where, $S[J]=\int d^{\nu} r\left[\frac{1}{2} \phi_{r}\left(-\nabla_{r}^{2}\right) \phi_{r}-J_{r} \phi_{r}+\int \frac{d \Omega}{\sqrt{2 \pi}} \tilde{V}(\Omega) e^{i \Omega \phi_{r}} e^{\frac{1}{2} \Omega^{2} I_{(1)}\left[M^{2}\right]}\right]$ with $\nabla_{r}$ the gradient with respect to $r$ in $\nu$-dimensional Euclidean space. Up to here, we have introduced the Coleman's normal-ordering prescription in the functional integral formalism. Actually, many years ago, the normal-ordered Hamiltonian of the sine-Gordon field theory has been used in the Euclidean functional integral formalism to show the equivalence between the sine-Gordon and massive Thirring field theories [9]. One will see that Eq.(11) will give rise to the same result in Ref. [2].

Now we calculate the GEP of Eq.(1) from Eq.(11) by using the procedure in Ref. [6]. For this purpose, $Z[J]$ will be modified through the following steps. First, a parameter $\mu$ is introduced by adding a vanishing term $\int d^{\nu} r \frac{1}{2} \phi_{r}\left(\mu^{2}-\right.$ $\left.\mu^{2}\right) \phi_{r}$ into $S[J]$. Then, shift $\phi_{r}$ to $\phi_{r}+\Phi$ with $\Phi$ a constant background field, i.e., $S[J] \rightarrow \int d^{\nu} r\left[\frac{1}{2} \phi_{r}\left(-\nabla_{r}^{2}+\mu^{2}\right) \phi_{r}-\right.$ $\left.J_{r} \phi_{r}-J_{r} \Phi+S_{D}\right]$ with $S_{D}=\int d^{\nu} r\left[-\frac{1}{2} \mu^{2} \phi_{r}^{2}+\int \frac{d \Omega}{\sqrt{2 \pi}} \tilde{V}(\Omega) e^{i \Omega\left(\phi_{r}+\Phi\right)} e^{\frac{1}{2} \Omega^{2} I_{(1)}\left[M^{2}\right]}\right]$. Thirdly, in the last resultant expression of $S[J]$, insert an expansion factor $\delta$ in front of $S_{D}$. Thus, $Z[J]$ is modified as the following $Z[J, \delta]$

$$
\begin{aligned}
Z[J, \delta]= & \exp \left\{\int d^{\nu} r\left[\frac{1}{2} I_{(0)}\left[M^{2}\right]-\frac{1}{2} M^{2} I_{(1)}\left[M^{2}\right]+J_{r} \Phi\right]\right\} \cdot \\
& \int \mathcal{D} \phi \exp \left\{-\int d^{\nu} r\left[\frac{1}{2} \phi_{r}\left(-\nabla_{r}^{2}+\mu^{2}\right) \phi_{r}-J_{r} \phi_{r}\right]\right\} \exp \left\{-\delta S_{D}\right\} \\
= & {\left[\operatorname{det}\left(-\nabla_{r}^{2}+\mu^{2}\right)\right]^{-\frac{1}{2}} \exp \left\{\int d ^ { \nu } r \left[\frac{1}{2} I_{(0)}\left[M^{2}\right]-\frac{1}{2} M^{2} I_{(1)}\left[M^{2}\right]+\frac{1}{2} \int d^{\nu} r_{1} J_{r} f_{r r_{1}}^{-1} J_{r_{1}}\right.\right.} \\
& \left.\left.+J_{r} \Phi\right]\right\} \frac{\int \mathcal{D} \phi \exp \left\{-\int d^{\nu} r\left[\frac{1}{2} \phi_{r}\left(-\nabla_{r}^{2}+\mu^{2}\right) \phi_{r}-J_{r} \phi_{r}\right]\right\} \exp \left\{-\delta S_{D}\right\}}{\int \mathcal{D} \phi \exp \left\{-\int d^{\nu} r\left[\frac{1}{2} \phi_{r}\left(-\nabla_{r}^{2}+\mu^{2}\right) \phi_{r}-J_{r} \phi_{r}\right]\right\}}
\end{aligned}
$$

where, $\operatorname{det}\left(-\nabla_{r}^{2}+\mu^{2}\right)$ is the determinant of $\left(-\nabla_{r}^{2}+\mu^{2}\right)$ and $f_{r r_{1}}^{-1}=\int \frac{d^{\nu} p}{(2 \pi)^{\nu}} \frac{1}{p^{2}+\mu^{2}} e^{i p \cdot\left(r-r_{1}\right)}$. In Eq.(13), the result of the Gaussian functional integral $\int \mathcal{D} \phi \exp \left\{-\int d^{\nu} r\left[\frac{1}{2} \phi_{r}\left(-\nabla_{r}^{2}+\mu^{2}\right) \phi_{r}-J_{r} \phi_{r}\right]\right\}=\left[\operatorname{det}\left(-\nabla_{r}^{2}+\right.\right.$ $\left.\left.\mu^{2}\right)\right]^{-\frac{1}{2}} \exp \left\{\frac{1}{2} \int d^{\nu} r d^{\nu} r_{1} J_{r} f_{r r_{1}}^{-1} J_{r_{1}}\right\}$ has been used. Correspondingly, $W[J]$ is modified as $W[J, \delta]$. It is evident that, extrapolating $W[J, \delta]$ to $\delta=1$, one recovers $W[J]$. After the above modifications, expanding the logarithm of the functional integral in $W[J, \delta]=\ln Z[J, \delta]$ as a series in $\delta$ (i.e., expanding first $e^{-\delta S_{D}}$ and then the logarithmic function), then truncating the series at the first order in $\delta$, and finally carrying out the functional integrations, one has

$$
\begin{aligned}
W[J, \delta]= & \int d^{\nu} r\left\{-\frac{1}{2}\left(I_{(0)}\left[\mu^{2}\right]-I_{(0)}\left[M^{2}\right]\right)-\frac{1}{2} M^{2} I_{(1)}\left[M^{2}\right]+J_{r} \Phi+\frac{1}{2} \int d^{\nu} r_{1} J_{r} f_{r_{1} r}^{-1} J_{r_{1}}\right. \\
& +\delta\left[\frac{1}{2} \mu^{2}\left[I_{(1)}\left[\mu^{2}\right]+\left(\int d^{\nu} r_{1} f_{r r_{1}}^{-1} J_{r_{1}}\right)^{2}\right]-\int_{-\infty}^{\infty} \frac{d \alpha}{\sqrt{\pi}} V\left(\alpha \sqrt{2\left(I_{(1)}\left[\mu^{2}\right]-I_{(1)}\left[M^{2}\right]\right)}\right.\right. \\
& \left.\left.+\int d^{\nu} r_{1} f_{r_{1} r}^{-1} J_{r_{1}}+\Phi\right) e^{\left.-\alpha^{2}\right]}\right]
\end{aligned}
$$

where, the first-order term of $\delta$ arises from the functional integral $\frac{\int \mathcal{D} \phi S_{D} \exp \left\{-\int d^{\nu} r\left[\frac{1}{2} \phi_{r}\left(-\nabla_{r}^{2}+\mu^{2}\right) \phi_{r}-J_{r} \phi_{r}\right]\right\}}{\int \mathcal{D} \phi \exp \left\{-\int d^{\nu} r\left[\frac{1}{2} \phi_{r}\left(-\nabla_{r}^{2}+\mu^{2}\right) \phi_{r}-J_{r} \phi_{r}\right]\right\}}$. In Eq. (14), we have used the integral formula $\int_{-\infty}^{\infty} \frac{d \alpha}{\sqrt{2 \pi}} e^{-\frac{\alpha^{2}}{2}+\sqrt{2 a} \alpha}=e^{a}$ and the result $\left[\operatorname{det}\left(-\nabla_{r}^{2}+\mu^{2}\right)\right]^{-\frac{1}{2}}=\exp \left\{-\frac{1}{2} \int d^{\nu} r I_{0}\left[\mu^{2}\right]\right\}$. Therefore, up to the first order of $\delta$, Eq.(8) gives 


$$
\begin{aligned}
\varphi_{r}= & \Phi+\int d^{\nu} r_{1} f_{r r_{1}}^{-1} J_{r_{1}}+\delta \mu^{2} \int d^{\nu} r_{1} d^{\nu} r_{2} f_{r r_{1}}^{-1} f_{r_{1} r_{2}}^{-1} J_{r_{2}} \\
& -\delta \int d^{\nu} r_{1} f_{r r_{1}}^{-1} \int_{-\infty}^{\infty} \frac{d \alpha}{\sqrt{\pi}} V^{(1)}\left(\alpha \sqrt{2\left(I_{(1)}\left[\mu^{2}\right]-I_{(1)}\left[M^{2}\right]\right)}+\int d^{\nu} r_{1} f_{r r_{1}}^{-1} J_{r_{1}}+\Phi\right) e^{-\alpha^{2}}
\end{aligned}
$$

where $V^{(n)}(\alpha)=\frac{d^{n} V(\alpha)}{(d \alpha)^{n}}=\int \frac{d \Omega}{\sqrt{2 \pi}} \tilde{V}(\Omega)(i \Omega)^{n} e^{i \Omega \alpha}$. In the last equation, one can take $\varphi_{r}=\varphi=\Phi$ 3 and hence solve it to get $J_{r}$ in terms of $\Phi$. This enforces $J_{r}$ to become a series in $\delta$ and vanish in the zeroth order of $\delta$ [ 6 ]. From Eqs.(14) and (9), one can see that $J_{r}$ truncated at the first order of $\delta$ has no contributions to $\mathcal{V}(\varphi)$ up to first order of $\delta$. Therefore, we have to take $J_{r}=0$ for truncating Eq.(9) at the first order of $\delta$, and obtain the following result

$$
\begin{aligned}
\mathcal{V}(\Phi)= & \frac{1}{2}\left(I_{(0)}\left[\mu^{2}\right]-I_{(0)}\left[M^{2}\right]\right)+\frac{1}{2} M^{2} I_{(1)}\left[M^{2}\right]-\frac{1}{2} \mu^{2} I_{(1)}\left[\mu^{2}\right] \\
& +\int_{-\infty}^{\infty} \frac{d \alpha}{\sqrt{\pi}} e^{-\alpha^{2}} V\left(\alpha \sqrt{2\left(I_{(1)}\left[\mu^{2}\right]-I_{(1)}\left[M^{2}\right]\right)}+\Phi\right) .
\end{aligned}
$$

Obviously, the above equation is dependent on the arbitrary parameter $\mu$. In accordance with the "principle of minimal sensitivity" [6, 10], $\mu$ can be determined by requiring that $\mu$ should minimize $\mathcal{V}(\varphi)$. The stationary condition, $\frac{\partial \mathcal{V}(\varphi)}{\partial \mu^{2}}=0$, yields

$$
\mu^{2}(\varphi)=\int_{-\infty}^{\infty} \frac{d \alpha}{\sqrt{\pi}} e^{-\alpha^{2}} V^{(2)}\left(\alpha \sqrt{2\left(I_{(1)}\left[\mu^{2}\right]-I_{(1)}\left[M^{2}\right]\right)}+\Phi\right),
$$

and the stability condition, $\frac{\partial^{2} \mathcal{V}(\varphi)}{\left(\partial \mu^{2}\right)^{2}} \geq 0$, gives rise to

$$
1+\frac{1}{4} I_{(2)}\left[\mu^{2}\right] \int_{-\infty}^{\infty} \frac{d \alpha}{\sqrt{\pi}} e^{-\alpha^{2}} V^{(4)}\left(\alpha \sqrt{2\left(I_{(1)}\left[\mu^{2}\right]-I_{(1)}\left[M^{2}\right]\right)}+\Phi\right) \geq 0 .
$$

In order to investigate the symmetry breaking phenomena, one usually needs another stationary point condition $\frac{d \mathcal{V}(\varphi)}{d \varphi}=0$. This condition yields the following equation

$$
\int_{-\infty}^{\infty} \frac{d \alpha}{\sqrt{\pi}} e^{-\alpha^{2}} V^{(1)}\left(\alpha \sqrt{2\left(I_{(1)}\left[\mu^{2}\right]-I_{(1)}\left[M^{2}\right]\right)}+\Phi\right)=0 .
$$

Noticing the equivalence between $I_{(n)}\left[Q^{2}\right]$ and $I_{n}\left[Q^{2}\right]$, and going back to the Minkowski space, Eq.(16) with Eqs.(17) and (18) will give the GEP of the system, Eq.(1). We note that it is identical to that in Ref. [2]. We observe that, here, no renormalization procedure is needed for the case of $D<3$, because the first three terms in Eq.(16) and $\left(I_{(1)}\left[\mu^{2}\right]-I_{(1)}\left[M^{2}\right]\right)$ are finite for $D<3$. As for the case of $D=3$, the first three terms in Eq.(16) and $\left(I_{(1)}\left[\mu^{2}\right]-I_{(1)}\left[M^{2}\right]\right)$ are divergent, and so Eqs.(16) - (19) have divergences. Hence, when $D=3$, the Coleman's normal-ordering prescription is not sufficient to renormalize the GEP, and further renormalization precedures are needed. In fact, the Coleman's normal-ordering prescription amounts just to renormalizing the mass parameter. For the case of $D=3$, one can further renormalize other model parameters and even the field to make the GEP finite.

By way of explanation and justification, we consider the $\lambda \phi^{4}$ field theory with the following potential

$$
V\left(\phi_{x}\right)=\frac{1}{2} m^{2} \phi_{x}^{2}+\frac{\lambda}{4} \phi_{x}^{4}
$$

Employing the formulae $\int_{-\infty}^{\infty} \alpha^{2 n} e^{-\alpha^{2}} d \alpha=2^{-n} \cdot 1 \cdot 3 \cdot 5 \cdots(2 n-1)$ and $\int_{-\infty}^{\infty} \alpha^{2 n+1} e^{-\alpha^{2}} d \alpha=0$, one can easily finish the ordinary integrations over $\alpha$ in Eqs.(16)-(19), and obtain

$$
\begin{aligned}
\mathcal{V}(\varphi)= & \frac{1}{2}\left(I_{(0)}\left[\mu^{2}\right]-I_{(0)}\left[M^{2}\right]\right)+\frac{1}{2} M^{2} I_{(1)}\left[M^{2}\right]-\frac{1}{2} \mu^{2} I_{(1)}\left[\mu^{2}\right] \\
& +\frac{1}{2} m^{2}\left(I_{(1)}\left[\mu^{2}\right]-I_{(1)}\left[M^{2}\right]+\Phi^{2}\right)+\frac{\lambda}{4}\left[\frac { 3 } { 4 } \left(2 I_{(1)}\left[\mu^{2}\right]\right.\right. \\
& \left.\left.-2 I_{(1)}\left[M^{2}\right]\right)^{2}+3\left(2 I_{(1)}\left[\mu^{2}\right]-2 I_{(1)}\left[M^{2}\right]\right) \Phi^{2}+\Phi^{4}\right],
\end{aligned}
$$

\footnotetext{
${ }^{3}$ Generally, different choices of $\varphi$ will give rise to an identical result. One can find a detailed discussion on this point in Appendix A of Ref. [6].
} 


$$
\mu^{2}=m^{2}+3 \lambda\left(I_{(1)}\left[\mu^{2}\right]-I_{(1)}\left[M^{2}\right]+\Phi^{2}\right)
$$

and

$$
\frac{d \mathcal{V}(\varphi)}{d \varphi}=\Phi\left(m^{2}+\frac{3 \lambda}{2}\left(2 I_{(1)}\left[\mu^{2}\right]-2 I_{(1)}\left[M^{2}\right]\right)+\lambda \Phi^{2}\right)=0 .
$$

Recalling $I_{(0)}\left[Q^{2}\right]=I_{0}\left[Q^{2}\right]$ (up to an infinite constant) and $2 I_{(1)}\left[Q^{2}\right]=I_{1}\left[Q^{2}\right]$, and noticing that for the case of $(1+1)$ dimensions, $\frac{1}{2}\left(I_{0}\left[\mu^{2}\right]-I_{0}\left[M^{2}\right]\right)+\frac{1}{4} M^{2} I_{1}\left[M^{2}\right]-\frac{1}{4} \mu^{2} I_{1}\left[\mu^{2}\right]=\frac{\mu^{2}-M^{2}}{8 \pi}$ as well as $\left(I_{1}\left[\mu^{2}\right]-I_{1}\left[M^{2}\right]\right)=-\frac{1}{2 \pi} \ln \frac{\mu^{2}}{M^{2}}$, one can find that Eq.(21) and Eq.(22) with $D=1$ are consistent, respectively, with Eqs.(A6) and (A7) for $B=0$ in Ref. [3] (Chang) (there, the normal-ordering mass $M$ was taken as $m$ and $m^{\prime}$ there corresponds to $\mu$ here) 1 . Furthermore, the renormalized mass and coupling can be calculated as

$$
\left.m_{R}^{2} \equiv \frac{d^{2} \mathcal{V}(\Phi)}{d \Phi^{2}}\right|_{\Phi=0}=m^{2}+3 \lambda\left(I_{(1)}\left[m_{R}^{2}\right]-I_{(1)}\left[M^{2}\right]\right)
$$

and

$$
\left.\lambda_{R} \equiv \frac{1}{3 !} \frac{d^{4} \mathcal{V}(\Phi)}{d \Phi^{4}}\right|_{\Phi=0}=\lambda \frac{1-6 \lambda I_{(2)}\left[m_{R}^{2}\right]}{1+3 \lambda I_{(2)}\left[m_{R}^{2}\right]},
$$

respectively. The above expression of $\lambda_{R}$ is consistent with Eq.(3.44) in Ref. [1] (1980) and Eq. (3.19) in Ref. [1] (1985), and has no explicit dependence upon the normal-ordering mass $M$ (just an implicit dependence upon $M$ through $m_{R}$ ). This fact implies that the Coleman's normal-ordering prescription is involved only in the renormalizaton of the mass parameter. Because the integral $I_{(2)}$ is finite for the case of $D<3$, the coupling does not require further renormalization procedure. Substituting Eq.(24) into Eqs.(21) and (22), one can get the GEP in terms of $m_{R}$ instead of $m$ and the resultant expressions for low dimensions are consistent with those in Ref. [1] (1985). Eq.(24) reflects the relation between $m$ and $M_{R}$, and has been discussed in detail for low dimensions in Ref. [11]. By the way, besides simplifying the renormalization procedure in low dimensions, the Coleman's normal-ordering prescription makes it possible to investigate the symmetry restoration phenomenon in quantum field theory [11, 12]. As for the case of $D=3$, both Eq.(24) and Eq.(25) are no longer finite relations, and further renormalization procedure will be needed to make the GEP finite. Stevenson and his collaborators have investigated this problem and proposed two non-trivial $\lambda \phi^{4}$ theories [13] [1] (1985). Based on the Coleman's normal-ordering prescription, one of the present authors, Lu, gave a further discussion about the Stevenson's two non-trivial $\lambda \phi^{4} \mathrm{~s}$ [14] (in Ref. [14], one can find many other references related to this problem).

In conclusion, we has demonstrated that the Coleman's normal-ordering prescription can be formally used in the functional integral formalism to renormalize the GEP for a class of system in low dimensions. This conclusion will also be valid for the finite temperature GEP [15, 16]. Before ending this paper, we point out that the above renormalizability is understandable from the viewpoint of Feynman diagrams. The Coleman's normal-ordering prescription can make ultraviolet divergences disappear in the theory whose primitively divergent graph is just the one-loop diagram with only one vertex. The $(1+1)$-dimensional scalar field theories without derivative interactions are just such ones [3]. Hence, the finiteness of Eqs.(16) - (19) with $D=1$ is conceivable. As for the case of $D=2$, the additional primitively divergent graphes are two- or multi-loop diagrams with multi-vertices. These additional divegent diagrams are not included in the GEP, because the GEP is just the sum of all possible cactus diagrams [17] [1](1980) (a cactus diagram consists of one-loop diagrams with multi-vertices and/or loop diagrams with one vertex). And so the GEP in $(2+1)$ dimensions can be made finite by the Coleman's normal-ordering prescription. However, unfortuately, when $D=3$, the one-loop diagram with two vertices, which comprises GEP, is divergent (for $D=2$, such a diagram is finite), and so the Coleman's normal-ordering prescription is not sufficient to make the $(3+1)$-dimensional GEP finite.

\section{ACKNOWLEDGMENTS}

Lu acknowledges Prof. J. H. Yee's helpful discussions. This project was supported by the Korea Research Foundation (99-005-D00011). Lu's work was also supported in part by the National Natural Science Foundation of China under the grant No. 19875034.

\footnotetext{
${ }^{4}$ The erratum for Eq.(A6) can be found in the page 1979 of Phys. Rev. D 16 (1977).

${ }^{5}$ Here, the definition of the renormalized coupling is slightly different from that in Ref. 化 $(1980,1985)$, because the coupling there is 4 times of the one here.
} 
[1] Schiff L I 1963 Phys. Rev. 130458

Cornwall J M, Jackiw R and Tomboulis E 1974 Phys. Rev. D 102428

Barnes T and Ghandour G I 1980 Phys. Rev. D 22924

Bardeen W A, Moshe M and Bander M 1984 Phys. Rev. Lett. 521188

Stevenson P M 1985 Phys. Rev. D 321389

[2] Lu W F, Chen S Q and Ni G J 1995 J. Phys. A 287233

[3] Coleman S 1975 Phys. Rev. D 112088

Chang S J 1976 Phys. Rev. D 132778

[4] Negele J W and Orland H 1988 Quantum Many-Particle System (New York : Addison-Wesley) Auerbach A 1994 Interacting Electrons and Quantum Magnetism (New York : Springer-Verlag)

[5] Okopińska A 1987 Phys. Rev. D 351835

You S K, Kim C K, Nahm K and Noh H S 2000 Phys. Rev. C 62045503

Lu W F, Kim C K, Yee J H and Nahm K 2001 Phys. Rev. D 64025006

[6] Stancu I and Stevenson P M 1990 Phys. Rev. D 422710

Stancu I 1991 Phys. Rev. D 431283

[7] Boccara N 1990 Functional Analysis - An Introduction for Physicists (New York : Academic)

[8] Ramond P 1990 Field Theory: A Modern Primer Revised Printing (New York : Addison-Wesley)

[9] Naón C M 1985 Phys. Rev. D 312035

[10] Stevenson P M 1981 Phys. Rev. D 232916

Kaufmann S K and Perez S M 1984 J. Phys. A 172027

Stevenson P M 1984 Nucl. Phys. B 23165

[11] Lu W F and Ni G J 1998 Commun. Theor. Phys. 30595

[12] Lu W F, Ni G J and Wang Z G 1998 J Phys. G 24673

[13] Stevenson P M and Tarrach R 1986 Phys. Lett. B 176436

[14] Lu W F 1999 Mod. Phys. Lett. A 141421

[15] Okopińska A 1987 Phys. Rev. D 362415

Hajj G A and Stevenson P M 1988 Phys. Rev. D 37413

Haugerud H and Ravndal F 1991 Phys. Rev. D 432736

[16] Roditi I 1986 Phys. Lett. B 17785

Lu W F 1999 J. Phys. A 32739 ; 2000 J. Phys. G 261187

[17] Chang S J 1975 Phys. Rev. D 121071 